\title{
Self-reported symptoms of depression, anxiety and stress in Portuguese primary school-aged children
}

\author{
Diogo Costa ${ }^{1 *}$ (D), Marina Cunha2, Cláudia Ferreira², Augusta Gama ${ }^{1,3}$, Aristides M. Machado-Rodrigues ${ }^{1,4}$, \\ Vítor Rosado-Marques ${ }^{1,5}$, Helena Nogueira ${ }^{1,6}$, Maria-Raquel G. Silva ${ }^{1,7}$ and Cristina Padez ${ }^{1}$
}

\begin{abstract}
Background: Symptoms of depression, anxiety and stress experienced during childhood might have a negative impact on development. This study explores factors associated with such symptoms among Portuguese primary school-aged children.

Methods: A sample of children ( $n=1022$, mean age $=8.77$ years old) was recruited in public and private schools from the cities of Coimbra, Lisbon and Porto, Portugal. The children's version of the Depression, Anxiety and Stress Scale (DASS-C) was self-administered. Multivariate logistic regression models were fitted to measure associations (expressed as Odds Ratio, OR and 95\% Confidence Intervals, Cl) between each DASS-C subscale, dichotomized by its 4th vs. 3rd or less quartiles (symptoms increase with scores), and covariates: child sex, age, socioeconomic status (SES), sports activity beyond school, children self-assessed health status, child and mother's body mass index and mother's DASS scores.

Results: Age was negatively associated with anxiety symptoms (adjusted OR, 95\% Cl: 0.70, 0.57-0.87) and girls, compared to boys, presented lower odds of depressive and stress symptoms (adjusted OR, 95\% Cl: 0.65, 0.47-0.92 and $0.57,0.41-0.80$, respectively). A low socioeconomic status was associated with more frequent symptoms of stress (adjusted OR, 95\%Cl for low compared to high SES: 1.61, 1.01-2.56). Children with poorer self-assessed health status and whose mothers scored higher in the DASS also presented significantly higher odds of scoring in the 4th quartile (vs. 3rd or less) of the three DASS-C subscales.
\end{abstract}

Conclusions: These results suggest the need to tailor preventive efforts targeting childhood mental health symptoms.

Keywords: Depression, Anxiety, Stress, Determinants, Children, Primary-school

\section{Background}

Childhood and adolescent mental health problems represent a growing public health concern that may have longlasting negative effects [1]. A previous meta-analysis of studies documenting the prevalence of mental disorders in childhood and adolescence, found a worldwide (27 countries) prevalence of $6.5 \%$ for any anxiety disorder (95\% Confidence Interval: 4.7-9.1), and of $2.6 \%$ for any depressive disorder (95\% Confidence Interval: 1.7-3.9) [2].

Across Europe, there is a substantial number of children with unmet needs for mental health care [3], although

\footnotetext{
* Correspondence: diogo.costa@uc.pt

${ }^{1}$ Research Centre for Anthropology and Health, Department of Life Sciences, University of Coimbra, Calçada Martim de Freitas, Edifício São Bento, 3000-456 Coimbra, Portugal

Full list of author information is available at the end of the article
}

studies documenting the magnitude and factors associated with the most common mental health problems, particularly in younger ages, are limited. There are no recently published prevalence estimates of mental health problems in such young ages in Portugal. There is, however, indication of an increase (by 23\%) in the number of children attending outpatient psychiatric consultations, between 2011 and 2013 in the country, and an increase of 30\% in the number of new consultations [4].

Depression and anxiety symptoms among young children can have a negative impact on their development $[5,6]$, affecting their quality of life [7] and constitute a risk factor for poor academic performance and poor social functioning [8].

Even at low thresholds, depressive and anxiety symptoms may represent an increased risk for the development of mental disorders in adulthood, particularly, 
depression [7, 9]. Several mental health problems observed during adulthood might have their origin in childhood and adolescence adverse experiences [10-12]. Longitudinal evidence also supports that social factors can play a significant role in determining mental health problems during childhood and adolescence [13].

Poverty, or a socioeconomically disadvantageous status, is a known risk factor for depression and anxiety symptoms among children [14-16]. Children whose mothers have been diagnosed with a major psychiatric disorder, also seem to present an increased risk for the development of mental problems [17], particularly for mother's major depression disorder $[18,19]$. Childhood obesity is another factor that might be associated with children mental health. Obesity may lead to depression in adolescents [20] and being overweighed or obese may be the leading reason for teasing and bullying among youth [21].

Mental health problems have also been associated with younger age groups (compared with adolescents) [22]. Mixed findings have been documented regarding sex, with evidence of more frequent mental problems occurring among boys [22] but also among girls [23].

Physical activity has also been associated with a lower likelihood of anxiety and depression symptoms [24]. However, studies using younger samples (including children) are needed, since the beneficial effect of physical activity over mental distress is not clear in younger ages [25].

Despite this knowledge, there is still need for better data gathering procedures and for the design of effective preventive actions tackling children mental health problems [26]. There are few studies involving reports of young children and considering several individual-level factors. Most studies depend on parents or other informants to assess child's emotional state and behavior, increasing the propensity for socially desirable responses.

This study aims to explore several individual-level factors associated with self-reported symptoms of depression, anxiety and stress in a large sample of Portuguese children.

\section{Methods}

\section{Participants and procedures}

This study is part of a larger project entitled "Inequalities in Childhood Obesity: the impact of the socioeconomic crisis in Portugal from 2009 to 2015". During 2016 and 2017, a total of 8472 school-aged children (mean age: 7.17 years, standard deviation: 1.91, 50.8\% male), were recruited from 118 schools, public and private, in the cities of Porto, Coimbra and Lisbon. Participation rates were $60 \%$ in Porto, $58 \%$ in Coimbra and $67 \%$ in Lisbon. The same schools that participated in two previous projects were included. These projects were conducted during 2002 to assess childhood obesity prevalence [27], and during 2009 to assess changes in the prevalence of childhood obesity and the obesogenic environment [28].

Children attending pre-primary (if available in the school) and primary school were eligible to participate. Information letters describing the objective of the study were disseminated to all parents and written informed consent was asked for their participation and of their child. To parents of children aged 7.5 years and older, a specific additional consent was asked to allow their participation in a school-based survey, corresponding to the self-administration of a standardized questionnaire. The sample analyzed in this study is comprised of those who completed these self-administered questionnaires.

\section{Parents}

Parents answered a standardized questionnaire comprising different sections that included: parental demographic and socioeconomic information, children lifestyle and parental common mental health symptoms.

For this study, father's educational level was used as a proxy measure of socioeconomic status (SES), and categorized in low (corresponding to parents that had 9 years of school completed or less), medium (corresponding to parents that had secondary school level or from 10 to 12 years of school completed) and high (parents that had a university degree).

Parents' self-reported height and weight was used to compute their Body Mass Index $\left(\mathrm{kg} / \mathrm{m}^{2}\right)$, which was subsequently categorized in normal, overweight or obese, according to the World Health Organization (WHO, 2000) guidelines for adults over 20 years old.

The adult version of the 21 item Portuguese Depression, Anxiety and Stress Scale - DASS [29, 30], was answered by the mother or the father (7 items for each subscale). Respondents were asked to refer to the previous week and provide their answer in a 4-option Likert scale (0 - Did not apply to me at all; 1 - Applied to me to some degree, or some of the time; 2 - Applied to me to a considerable degree, or a good part of time; 3 - Applied to me very much, or most of the time) regarding each item, which are statements representing specific negative emotional symptoms. Items are summed for each subscale, thus scores can vary between 0 and 21 , with higher scores indicative of more symptoms.

\section{Children}

Children date of birth and sex were reported by their parents and age was computed in years at the time of data collection.

Parents also answered whether their child participated in any organized (programmed and regular) sport activity other than the physical activity practiced at school (categorized as "yes/no"). 
Children's height and weight were objectively measured by trained researchers at school, in the morning, (SECA 220 scale and SECA 200 stadiometer) and BMI (as $\mathrm{kg} / \mathrm{m}^{2}$ ) was subsequently categorized according to the International Obesity Task Force (IOTF) [31] cut-off points in normal weight, children with overweight and children with obesity.

Children aged 7.5 years or older, whose parents consented, were asked to answer a set of self-administered tools. The first question of the KIDSCREEN-27 questionnaire [32] was used as a measure of self-assessed health status - "In general, how would you describe your health: Excellent, Very Good, Good, Bad, Very Bad" dichotomized in "Excellent or Very Good" and "Good, Bad or Very Bad" (only 0.5\% of children reported "Bad" or "Very Bad" health status, hence this dichotomization).

Children were also asked to answer the child version of the 21-item DASS (the DASS-C) [33]. Conceptually, this questionnaire follows the exact same principles of the adult version, with language modifications to the items so these can be comprehended by children. The Portuguese version of the DASS-C has been validated using a student sample [34]. The questionnaires were handed over to teachers who, in turn, distributed them to children with parental permission to respond. Prior to the delivery of the questionnaires they were informed about each question by the researchers. All children with parental consent answered the questionnaire individually in the classroom. The teachers could enlighten the children about the understanding of each question if the child requested. Only questionnaires filled out by children, individually, in the school were accepted.

\section{Ethical approval}

The study protocol was approved by Direção Geral do Ensino (Portuguese Ministry of Education) and Comissão Nacional de Proteção de Dados (CNPD), the Portuguese Data Protection Authority. All procedures were in accordance with the ethical standards of the Portuguese Data Protection Authority (Authorization number 745/ 2017) and with the 1964 Helsinki declaration and its later amendments or comparable ethical standards.

Written informed consent was obtained from children's parents.

\section{Data analysis}

Psychometric analysis of the DASS and DASS-C were conducted and are presented as Supplementary material (Tables 1, 2 and 3).

Out of the 8472 children assessed, 3957 were 7.5 years of age or older and 1469 provided full answers to the DASS-C questionnaire. A further 447 cases were excluded from the current analysis due to missing values in SES, mother BMI, DASS, if the DASS was not answered by the mother (mothers answered the DASS in more than $87 \%$ of cases), sports activity beyond that practiced at school and selfassessed health status. A total of 1022 cases were analyzed.

Compared to excluded children (Supplementary material - Table 4), those included in analysis presented a slightly higher mean age (8.77 vs. $8.62, p<0.001)$, were more frequently females $(52.9 \%$ vs. $47.5 \%, p=0.003)$, were more frequently from Coimbra $(53.7 \%$ of all included), practiced sport activity beyond school more often $(72.3 \%$ vs. $61.2 \%, \mathrm{p}<0.001)$ and their mothers presented lower mean scores in the DASS Anxiety subscale (1.16 vs. $1.42, p=0.009)$. Children classified with high socioeconomic status, were also more frequent among those included in analysis compared to those excluded $(p=0.005)$, although the proportions did not differ more than $5 \%$ between groups (Low $=24.5 \%$ vs. $28.7 \%$; Medium $=36.8 \%$ vs. $37.9 \%$; High $=38.7 \%$ vs. $33.3 \%$ ). Due to these differences, it is not possible to state that this group of children constitutes a representative sample of Portuguese school-aged children.

Counts (and proportions), means (and standard deviations, sd) and medians (interquartile range, IQR) were calculated to describe categorical and continuous variables.

Each subscale of the DASS-C was categorized according to the quartiles of their distribution. Due to granularity in the data, the tertiles of the DASS subscales distribution were used for categorizing mothers' DASS scores. Mean age was compared across the quartiles of the DASS-C subscales using ANOVA and the proportions of all other characteristics (sex, city of residence, children and mother's BMI categories, socioeconomic status, sport activity beyond school, self-assessed health status and mother's DASS tertiles scores) were compared across the quartiles of the DASS-C subscales using Chi-squared tests.

Then, each DASS-C subscale was dichotomized in quartile 4 (Q4) and quartiles 3, 2 or 1 (Q3, Q2 or Q1). The dichotomized variables were used as dependent variables in logistic regression (predicting the probability of scoring in Q4 vs. Q3, Q2 or Q1). Models were fitted for each individual variable and then fully adjusted. Crude and adjusted odds ratios (OR and AOR) and respective $95 \%$ confidence intervals $(95 \% \mathrm{CI})$ were computed as measures of the magnitude of associations.

\section{Results}

The psychometric analysis conducted to the DASS and DASS-C is presented as Supplementary material. In summary, the three solution factor (eigenvalue $>1$ ) was confirmed for the DASS and also for the DASS-C. Cronbach's Alphas for Depression, Anxiety and Stress subscales of the DASS in this sample were $0.84,0.81$ and 0.88 , respectively, and the corresponding values for the DASS-C were $0.75,0.73$ and 0.78 , respectively. 
The sample characteristics are presented in Table 1. The mean age of the 1022 children analyzed was 8.77 years old (ranging from 7.5 to 11.5 years) and $52.9 \%$ were girls. The prevalence of children with overweight was $18.9 \%$ and there were $5.2 \%$ of children with obesity. Considering mother's BMI categories, $24.1 \%$ were overweighed (without obesity) and $8.9 \%$ were obese. A total of $38.7 \%$ of fathers had a university degree (high socioeconomic status), and $72.3 \%$ of children practiced some type of organized sport activity (in a club or sports association) other than the physical activity practiced at school.

Regarding their self-assessed health status, $78.7 \%$ of children declared their health was Excellent or Very Good, while the remaining 21.3\% classified their health as Good, Bad or Very Bad (0.5\% reported "Bad" or "Very Bad").

Children mean (sd) scores in the DASS subscales were 2.35 (2.87) for Depressive symptoms, 2.01 (2.63) for Anxiety and 2.84 (3.09) for Stress. For mothers, these mean scores were 1.45 (2.41), 1.16 (2.11) and 3.87 (3.31), respectively.

Tables 2, 3 and 4 present the distribution of the children DASS-C Depressive, Anxiety and Stress scores, categorized in quartiles (higher scores correspond to most marked symptoms) according to the sample characteristics.

For the three DASS-C subscales quartiles, a statistically significant difference in the distribution of the sample proportions was found according to sex, city of residence, distribution of children self-assessed health status and distribution of the tertiles of mother's DASS subscales score. There were slightly more girls than boys in the 1st quartile of the three DASS-C subscales and the opposite in the 4th quartile. Children from Lisbon (compared to Coimbra and Porto) scored more frequently in the 3rd and 4th quartiles of the three DASSC subscales. A higher proportion of children reporting their health as Good, Bad or Very Bad was observed in the 4th quartile of the three DASS-C subscales. The proportion of the 3rd tertile of mother's DASS answers was higher in the 4th quartile of children DASS-C subscales for the three subscales.

Table 5 shows the results from the logistic regression models fitted. After adjustment, age was negatively associated with increased DASS-C Anxiety symptoms score (AOR, 95\%CI - Adjusted Odd Ratio, 95\% Confidence Interval: $0.70,0.57-0.87$ for scoring in the 4 th quartile vs. 3rd quartile or less). Compared with boys, girls presented lower odds of scoring in the 4th quartile of the DASS-C Depressive and Stress symptoms subscales, in the adjusted models (AOR, 95\%CI: 0.65, 0.47-0.82 and 0.57, 0.41-0.80, respectively). Compared with Coimbra, children from Lisbon (but not from Porto) were more likely to report depressive, anxiety and stress symptoms (AOR, 95\%CI: 1.75, 1.23-2.50; 1.55, 1.11-2.18; 2.16, $1.52-3.09$, respectively).
A low socioeconomic status, compared with a high socioeconomic status, was significantly associated with increased odds of scoring in the 4th quartile for the DASS-C Stress subscale (AOR, 95\%CI: 1.61, 1.01-2.56).

Children who self-assessed their health as Good, Bad or Very Bad, compared with those assessing their health as Excellent or Very Good, showed significantly higher odds of scoring in the 4th quartile of the three DASS-C symptoms subscales, after adjustment (AOR, 95\%CI for the DASS-C Depressive, Anxiety and Stress: 2.70, 1.87$3.91 ; 1.84,1.28-2.65 ; 1.96,1.34-2.88$ ).

Increased odds of scoring in the 4th quartile (vs. 3rd or less) of the DASS-C Depressive, Anxiety and Stress subscales were found for the 3rd tertiles of the distribution of the mother's scores (vs. 1st tertile) in the DASS Depressive, Anxiety and Stress subscales.

\section{Discussion}

This exploration of the factors associated with mental health symptoms of young children suggests that girls, compared to boys, were less likely to report depression and stress symptoms and that children assessing their health as only Good, Bad or Very Bad, compared with those assessing their health as Excellent or Very Good, showed more frequent symptoms of depression, anxiety and stress. Children' symptoms were also more frequent with the increase in mothers' own reports on depressive, anxiety and stress symptoms.

\section{Interpretation of findings \\ Sex and age}

The current findings suggest that boys tend to report higher levels of anxiety and depressive symptoms than girls. This has also been suggested in a recent Iranian study where more common mental health problems were found among boys [22]. However, in a large community sample of primary school aged children (8-12 years) from Singapore $(n=1655)$, girls reported more anxiety and depressive symptoms than boys [23]. Sex differences in the expression of these symptoms might be influenced by the cultural context, and might be less noticeable in younger age groups, thus becoming more pronounced with increasing age.

In a meta-analytic study of gender differences in emotion expression of children, it was found that girls show more positive emotions than boys during childhood, while boys show more externalizing emotions [35]. These differences were context-dependent for internalizing, externalizing and negative emotion expressions, and may depend on different tendencies of boys and girls to provide answers that conform to societal gender roles [35]. In our sample, such tendency might have influenced girls' responses and explain the observed sex-differences. 
Table 1 Sample characteristics $(n=1022)$

\begin{tabular}{|c|c|c|c|}
\hline & $n(\%)$, or Mean (sd) & Median (IQR) & Min-Max \\
\hline Age (years) & $8.77(0.77)$ & $9.00(1.50)$ & $7.5-11.5$ \\
\hline \multicolumn{4}{|c|}{ Sex } \\
\hline Boys & $481(47.1)$ & & \\
\hline Girls & $541(52.9)$ & & \\
\hline \multicolumn{4}{|l|}{ City of residence } \\
\hline Coimbra & $549(53.7)$ & & \\
\hline Lisbon & $352(34.4)$ & & \\
\hline Porto & $121(11.8)$ & & \\
\hline \multicolumn{4}{|l|}{ Children BMI (IOTF cut-offs) } \\
\hline Normal & $776(75.9)$ & & \\
\hline Overweight & $193(18.9)$ & & \\
\hline Obesity & $53(5.2)$ & & \\
\hline \multicolumn{4}{|l|}{ Mother BMI (WHO cut-offs) } \\
\hline Normal & $685(67.0)$ & & \\
\hline Overweight & $246(24.1)$ & & \\
\hline Obesity & $91(8.9)$ & & \\
\hline \multicolumn{4}{|l|}{ Socioeconomic status ${ }^{a}$ (Father education) } \\
\hline Low & $250(24.5)$ & & \\
\hline Medium & $376(36.8)$ & & \\
\hline High & $396(38.7)$ & & \\
\hline \multicolumn{4}{|l|}{ Sport Activity beyond school } \\
\hline Yes & $739(72.3)$ & & \\
\hline No & $283(27.7)$ & & \\
\hline \multicolumn{4}{|l|}{ Self Assessed Health Status } \\
\hline Excellent, Very good & $804(78.7)$ & & \\
\hline Good, Bad, Very Bad & $218(21.3)$ & & \\
\hline Children DASS Depressive symptoms score & $2.35(2.87)$ & $1.00(3.25)$ & $0-20$ \\
\hline Children DASS Anxiety symptoms score & $2.01(2.63)$ & $1.00(3.00)$ & $0-18$ \\
\hline Children DASS Stress symptoms score & $2.84(3.09)$ & $2.00(4.00)$ & $0-16$ \\
\hline Mother DASS Depressive symptoms score & $1.45(2.41)$ & $0.00(2.00)$ & $0-19$ \\
\hline Mother DASS Anxiety symptoms score & $1.16(2.11)$ & $0.00(1.00)$ & $0-18$ \\
\hline Mother DASS Stress symptoms score & $3.87(3.31)$ & $3.00(5.00)$ & $0-21$ \\
\hline
\end{tabular}

aLow socioeconomic status - if the father had 9 years of completed schooling or less; Medium socioeconomic status: if the father had between 10 and 12 years of completed schooling; High socioeconomic status - if the father had a university degree; DASS Depression, Anxiety and Stress scale, IOTF International obesity taskforce, WHO World Health Organization, sd Standard deviation, IRQ Interquartile range;

\section{Place of residence}

Children from Lisbon reported symptoms of common mental health more frequently than children from Coimbra or Porto. There may be specific features of Lisbon's urban environment which increase children likelihood of depressive, anxiety and stress symptoms. Lisbon metropolitan area is the country's most densely populated region, where people travel longer daily distances. One hypothesis that could explain these results is that in Lisbon children spend more time in traffic to and from school, which can affect negatively both mother and child anxiety, depressive and stress symptoms. Further comparisons within and across cities are needed to disentangle the individual effect of environmental characteristics on mental health symptoms of children, including the time commuting to school. Future studies should also consider the effect of living in a disadvantaged or deprived neighborhood, since it has been associated with poor mental health outcomes [36]. 
Table 2 Depressive symptoms subscale quartiles distribution according to sample characteristics

\begin{tabular}{|c|c|c|c|c|c|}
\hline \multirow[b]{3}{*}{$n$} & \multicolumn{5}{|c|}{ DASS-C Depressive symptoms subscale score } \\
\hline & $\mathrm{Q} 1(<=0.00)$ & Q2 (1.00-1.00) & Q3 (2.00-4.00) & Q4 $(5.00+)$ & $p$ \\
\hline & 352 & 178 & 307 & 185 & \\
\hline Age (continuous) [mean (sd)] & $8.80(0.72)$ & $8.85(0.79)$ & $8.74(0.78)$ & $8.72(0.81)$ & 0.255 \\
\hline \multicolumn{6}{|l|}{$\operatorname{Sex}[n(\%)]$} \\
\hline Boys & $143(29.7)$ & $82(17.0)$ & $153(31.8)$ & $103(21.4)$ & \\
\hline Girls & $209(38.6)$ & $96(17.7)$ & $154(28.5)$ & $82(15.2)$ & 0.006 \\
\hline \multicolumn{6}{|l|}{ City of residence $[n(\%)]$} \\
\hline Coimbra & $225(41.0)$ & $88(16.0)$ & $151(27.5)$ & $85(15.5)$ & $<0.001$ \\
\hline Lisbon & $81(23.0)$ & $67(19.0)$ & $119(33.8)$ & $85(24.1)$ & \\
\hline Porto & $46(38.0)$ & $23(19.0)$ & $37(30.6)$ & $15(12.4)$ & \\
\hline \multicolumn{6}{|c|}{ Children BMI (IOTF cut-offs) [n(\%)] } \\
\hline Normal & $270(34.8)$ & $136(17.5)$ & $237(30.5)$ & $133(17.1)$ & \\
\hline Overweight & $62(32.1)$ & $37(19.2)$ & $56(29.0)$ & $38(19.7)$ & \\
\hline Obesity & $20(37.7)$ & $5(9.4)$ & $14(26.4)$ & $14(26.4)$ & 0.442 \\
\hline \multicolumn{6}{|c|}{ Mother BMI (WHO cut-offs)[n(\%)] } \\
\hline Normal & $242(35.3)$ & $118(17.2)$ & $212(30.9)$ & $113(16.5)$ & \\
\hline Overweight & $83(33.7)$ & $45(18.3)$ & $71(28.9)$ & $47(19.1)$ & \\
\hline Obesity & $27(29.7)$ & $15(16.5)$ & $24(26.4)$ & $25(27.5)$ & 0.303 \\
\hline \multicolumn{6}{|c|}{ Socioeconomic status ${ }^{a}$ (Father education) $[\mathrm{n}(\%)]$} \\
\hline Low & 79 (31.6) & $33(13.2)$ & $85(34.0)$ & $53(21.2)$ & \\
\hline Medium & $133(35.4)$ & $66(17.6)$ & $106(28.2)$ & $71(18.9)$ & \\
\hline High & $140(35.4)$ & 79 (19.9) & $116(29.3)$ & $61(15.4)$ & 0.141 \\
\hline \multicolumn{6}{|c|}{ Sport Activity beyond school [n(\%)] } \\
\hline Yes & $264(35.7)$ & $125(16.9)$ & $226(30.6)$ & $124(16.8)$ & \\
\hline No & $88(31.1)$ & $53(18.7)$ & $81(28.6)$ & $61(21.6)$ & 0.212 \\
\hline \multicolumn{6}{|l|}{ Self Assessed Health Status [n(\%)] } \\
\hline Excellent, Very good & $304(37.8)$ & $146(18.2)$ & $240(29.9)$ & $114(14.2)$ & \\
\hline Good, Bad, Very Bad & $48(22.0)$ & $32(14.7)$ & $67(30.7)$ & $71(32.6)$ & $<0.001$ \\
\hline \multicolumn{6}{|c|}{ Mother DASS Depression score [n(\%)] } \\
\hline $\mathrm{T} 1(<=0.00)$ & $200(38.9)$ & $90(17.5)$ & $147(28.6)$ & $77(15.0)$ & \\
\hline T2 (1.00-1.00) & $67(33.3)$ & $33(16.4)$ & $62(30.8)$ & $39(19.4)$ & \\
\hline $\mathrm{T} 3(2.00+)$ & $85(27.7)$ & $55(17.9)$ & 98 (31.9) & $69(22.5)$ & 0.027 \\
\hline
\end{tabular}

DASS-C Depression, Anxiety and Stress scale - Children version; $\mathrm{p}-\mathrm{p}$-value from ANOVA (age) and Chi-square tests, sd Standard deviation, BMI Body Mass Index, IOTF International Obesity Taskforce, WHO World Health Organization, Q1, Q2, Q3, Q4 - Quartiles 1, 2, 3 and 4 of the DASS-C subscale score distribution; T1, T2, T3 - Tertiles 1,2 and 3 of the DASS-C subscale score distribution

a Low socioeconomic status - if the father had 9 years of completed schooling or less; Medium socioeconomic status: if the father had between 10 and 12 years of completed schooling; High socioeconomic status - if the father had a university degree;

\section{Body mass index}

A significant association between children and mother's BMI categories with depression, anxiety and stress symptoms was not found in this study. However, the point estimates obtained, particularly for mother's BMI, suggest a graded influence on child symptoms. Given the association of mother's BMI on children BMI $[37,38]$, the physical health burden associated with overweight and obesity [39], and the potential bullying and stigma experienced by overweight/obese children [21], interventions aiming to promote mental health among children should include strategies that also aim to prevent excessive weight gain.

\section{Socioeconomic status}

The consistent association found between parental socioeconomic status and children mental health, suggests that children of less educated fathers reported more frequent symptoms of common mental problems. This is in line with the results from other studies [14-16]. Growing up in a disadvantaged environment has been 
Table 3 Anxiety symptoms subscale quartiles distribution according to sample characteristics

\begin{tabular}{|c|c|c|c|c|c|}
\hline \multirow[b]{3}{*}{$n$} & \multicolumn{5}{|c|}{ DASS-C Anxiety symptoms subscale score } \\
\hline & $\mathrm{Q} 1(<=0.00)$ & Q2 (1.00-1.00) & Q3 (2.00-3.00) & Q4 $(4.00+)$ & $p$ \\
\hline & 381 & 202 & 228 & 211 & \\
\hline Age (continuous) [mean (sd)] & $8.81(0.73)$ & $8.83(0.79)$ & $8.78(0.81)$ & $8.65(0.80)$ & 0.053 \\
\hline \multicolumn{6}{|l|}{$\operatorname{Sex}[n(\%)]$} \\
\hline Boys & $160(33.3)$ & $93(19.3)$ & $117(24.3)$ & $111(23.1)$ & \\
\hline Girls & $221(40.9)$ & $109(20.1)$ & $111(20.5)$ & $100(18.5)$ & 0.041 \\
\hline \multicolumn{6}{|l|}{ City of residence $[n(\%)]$} \\
\hline Coimbra & $239(43.5)$ & $110(20.0)$ & $96(17.5)$ & $104(18.9)$ & $<0.001$ \\
\hline Lisbon & $90(25.6)$ & $70(19.9)$ & $101(28.7)$ & $91(25.9)$ & \\
\hline Porto & $52(43.0)$ & $22(18.2)$ & $31(25.6)$ & $16(13.2)$ & \\
\hline \multicolumn{6}{|c|}{ Children BMI (IOTF cut-offs) [n(\%)] } \\
\hline Normal & $293(37.8)$ & $160(20.6)$ & $170(21.9)$ & $153(19.7)$ & \\
\hline Overweight & $69(35.8)$ & $35(18.1)$ & $44(22.8)$ & $45(23.3)$ & \\
\hline Obesity & $19(35.8)$ & $7(13.2)$ & $14(26.4)$ & $13(24.5)$ & 0.710 \\
\hline \multicolumn{6}{|c|}{ Mother BMI (WHO cut-offs) [n(\%)] } \\
\hline Normal & $274(40.0)$ & $134(19.6)$ & $152(22.2)$ & $125(18.2)$ & \\
\hline Overweight & $80(32.5)$ & 49 (19.9) & $57(23.2)$ & $60(24.4)$ & \\
\hline Obesity & $27(29.7)$ & $19(20.9)$ & $19(20.9)$ & $26(28.6)$ & 0.093 \\
\hline \multicolumn{6}{|c|}{ Socioeconomic status ${ }^{a}$ (Father education) $[\mathrm{n}(\%)]$} \\
\hline Low & $83(33.2)$ & $43(17.2)$ & $66(26.4)$ & $58(23.2)$ & \\
\hline Medium & $147(39.1)$ & $73(19.4)$ & $72(19.1)$ & $84(22.3)$ & \\
\hline High & $151(38.1)$ & $86(21.7)$ & $90(22.7)$ & $69(17.4)$ & 0.123 \\
\hline \multicolumn{6}{|c|}{ Sport Activity beyond school [n(\%)] } \\
\hline Yes & $287(38.8)$ & $148(20.0)$ & $165(22.3)$ & $139(18.8)$ & \\
\hline No & $94(33.2)$ & $54(19.1)$ & $63(22.3)$ & $72(25.4)$ & 0.103 \\
\hline \multicolumn{6}{|c|}{ Self Assessed Health Status [n(\%)] } \\
\hline Excellent, Very good & $322(40.0)$ & $167(20.8)$ & $172(21.4)$ & $143(17.8)$ & \\
\hline Good, Bad, Very Bad & $59(27.1)$ & $35(16.1)$ & $56(25.7)$ & $68(31.2)$ & $<0.001$ \\
\hline \multicolumn{6}{|c|}{ Mother DASS Anxiety score [n(\%)] } \\
\hline $\mathrm{T} 1(<=0.00)$ & $254(44.4)$ & $108(18.9)$ & $107(18.7)$ & $103(18.0)$ & \\
\hline T2 (1.00-1.00) & $65(31.6)$ & $50(24.3)$ & $55(26.7)$ & $36(17.5)$ & \\
\hline $\mathrm{T} 3(2.00+)$ & $62(25.4)$ & 44 (18.0) & $66(27.0)$ & 72 (29.5) & $<0.001$ \\
\hline
\end{tabular}

DASS-C Depression, Anxiety and Stress scale - Children version, $p$ p-value from ANOVA (age) and Chi-square tests, sd Standard deviation, BMI Body Mass Index, IOTF International Obesity Taskforce, WHO World Health Organization; Q1, Q2, Q3, Q4 - Quartiles 1, 2, 3 and 4 of the DASS-C subscale score distribution; T1, T2, T3 - Tertiles 1,2 and 3 of the DASS-C subscale score distribution

a Low socioeconomic status - if the father had 9 years of completed schooling or less; Medium socioeconomic status: if the father had between 10 and 12 years of completed schooling; High socioeconomic status - if the father had a university degree;

associated with several poor health outcomes [40], thus, this particular result highlights the need to address socioeconomic disparities in Portuguese children from an early stage.

\section{Sport activity}

We did not find a significant association between mental health symptoms and sport activity outside school, although physical activity has been identified as a protective factor of poor mental health [41, 42]. Physical activity may act through physiological changes (decreases in cortisol levels) [43], psychological changes (e.g. reducing anger, fatigue) [44] and social changes (e.g. enabling opportunities for social support), thus reducing or preventing anxiety and depression symptoms. More detailed information about sports activity engagement among Portuguese primary-school aged children is needed to measure its effect on mental health. Nevertheless, given the documented benefits for health and the fact that Portuguese children in this age range present 
Table 4 Stress symptoms subscale quartiles distribution according to sample characteristics

\begin{tabular}{|c|c|c|c|c|c|}
\hline \multirow[b]{3}{*}{$n$} & \multicolumn{5}{|c|}{ DASS-C Stress symptoms subscale score } \\
\hline & $\mathrm{Q} 1(<=0.00)$ & Q2 (1.00-2.00) & Q3 (3.00-5.00) & Q4 $(6.00+)$ & $p$ \\
\hline & 300 & 278 & 257 & 187 & \\
\hline Age (continuous) [mean (sd)] & $8.86(0.72)$ & $8.78(0.75)$ & $8.71(0.86)$ & $8.70(0.77)$ & 0.074 \\
\hline \multicolumn{6}{|l|}{$\operatorname{Sex}[n(\%)]$} \\
\hline Boys & $118(24.5)$ & $113(23.5)$ & $140(29.1)$ & $110(22.9)$ & \\
\hline Girls & $182(33.6)$ & $165(30.5)$ & $117(21.6)$ & $77(14.2)$ & $<0.001$ \\
\hline \multicolumn{6}{|l|}{ City of residence [n(\%)] } \\
\hline Coimbra & $180(32.8)$ & $165(30.1)$ & $124(22.6)$ & $80(14.6)$ & $<0.001$ \\
\hline Lisbon & $77(21.9)$ & $79(22.4)$ & $104(29.5)$ & $92(26.1)$ & \\
\hline Porto & $43(35.5)$ & $34(28.1)$ & $29(24.0)$ & $15(12.4)$ & \\
\hline \multicolumn{6}{|c|}{ Children BMI (IOTF cut-offs) [n(\%)] } \\
\hline Normal & $224(28.9)$ & $230(29.6)$ & $186(24.0)$ & $136(17.5)$ & \\
\hline Overweight & $62(32.1)$ & $37(19.2)$ & $53(27.5)$ & $41(21.2)$ & \\
\hline Obesity & $14(26.4)$ & $11(20.8)$ & $18(34.0)$ & $10(18.9)$ & 0.075 \\
\hline \multicolumn{6}{|c|}{ Mother BMI (WHO cut-offs) [n(\%)] } \\
\hline Normal & $212(30.9)$ & $180(26.3)$ & $171(25.0)$ & $122(17.8)$ & \\
\hline Overweight & $70(28.5)$ & $76(30.9)$ & $57(23.2)$ & $43(17.5)$ & \\
\hline Obesity & $18(19.8)$ & $22(24.2)$ & $29(31.9)$ & $22(24.2)$ & 0.164 \\
\hline \multicolumn{6}{|c|}{ Socioeconomic status ${ }^{a}$ (Father education) $[\mathrm{n}(\%)]$} \\
\hline Low & $70(28.0)$ & $61(24.4)$ & $63(25.2)$ & $56(22.4)$ & \\
\hline Medium & $114(30.3)$ & $106(28.2)$ & $85(22.6)$ & $71(18.9)$ & \\
\hline High & $116(29.3)$ & $111(28.0)$ & $109(27.5)$ & $60(15.2)$ & 0.269 \\
\hline \multicolumn{6}{|c|}{ Sport Activity beyond school [n(\%)] } \\
\hline Yes & $228(30.9)$ & $203(27.5)$ & $183(24.8)$ & $125(16.9)$ & \\
\hline No & $72(25.4)$ & $75(26.5)$ & $74(26.1)$ & $62(21.9)$ & 0.168 \\
\hline \multicolumn{6}{|l|}{ Self Assessed Health Status [n(\%)] } \\
\hline Excellent, Very good & $258(32.1)$ & $230(28.6)$ & $191(23.8)$ & $125(15.5)$ & \\
\hline Good, Bad, Very Bad & $42(19.3)$ & $48(22.0)$ & $66(30.3)$ & $62(28.4)$ & $<0.001$ \\
\hline \multicolumn{6}{|l|}{ Mother DASS Stress score [n(\%)] } \\
\hline $\mathrm{T} 1(<=2.00)$ & $161(38.8)$ & $117(28.2)$ & $84(20.2)$ & $53(12.8)$ & \\
\hline T2 (3.00-5.00) & $78(25.1)$ & $86(27.7)$ & $95(30.5)$ & $52(16.7)$ & \\
\hline $\mathrm{T} 3(6.00+)$ & $61(20.6)$ & $75(25.3)$ & $78(26.4)$ & $82(27.7)$ & $<0.001$ \\
\hline
\end{tabular}

DASS-C Depression, Anxiety and Stress scale - Children version; $p$ p-value from ANOVA (age) and Chi-square tests, sd Standard deviation, BMI Body Mass Index, IOTF International Obesity Taskforce, WHO World Health Organization; Q1, Q2, Q3, Q4 - Quartiles 1, 2, 3 and 4 of the DASS-C subscale score distribution; T1, T2, T3 - Tertiles 1,2 and 3 of the DASS-C subscale score distribution

aLow socioeconomic status - if the father had 9 years of completed schooling or less; Medium socioeconomic status: if the father had between 10 and 12 years of completed schooling; High socioeconomic status - if the father had a university degree;

low levels of physical activity [45], sport activity should be promoted in early school years.

\section{Mother's mental health symptoms}

The influence of mother's depressive symptoms on children's negative emotions has been a topic of great concern [46, 47], but less is known regarding anxiety and stress, although anxiety is considered a very common condition in pre-adolescent ages [6].
A recent study in Uganda showed that caregiver anxiety, depressive symptoms and low social support, were associated with worse well-being in school-aged and adolescent children [48]. Depressive symptoms in mothers might limit their functioning, increase children's behavioral and developmental problems $[49,50]$.

Mother's negative emotions may influence child emotional and behavioral adjustment at very early stages, and a bidirectional influence (with the child's own symptoms influencing mother's emotion) might explain the congruence between 
Table 5 Factors associated with the DASS-C Depression, Anxiety and Stress subscales scores (dichotomized in Quartile 4 vs. Quartiles 3,2 or 1 of the score distribution of each subscale)

\begin{tabular}{|c|c|c|c|c|c|c|c|}
\hline \multirow[b]{2}{*}{$n$} & & \multicolumn{2}{|c|}{$\begin{array}{l}\text { DASS-C Depressive } \\
\text { Q4 }(n=185) \text { vs. }<\text { Q3 }(n=837)\end{array}$} & \multicolumn{2}{|c|}{$\begin{array}{l}\text { DASS-C Anxiety } \\
\text { Q4 }(n=211) \text { vs. }<\text { Q3 }(n=811)\end{array}$} & \multicolumn{2}{|c|}{$\begin{array}{l}\text { DASS-C Stress } \\
\text { Q4 }(n=187) \text { vs. }<\mathrm{Q} 3(n=835)\end{array}$} \\
\hline & & OR $(95 \% \mathrm{Cl})$ & AOR $(95 \% \mathrm{Cl})$ & OR $(95 \% \mathrm{Cl})$ & AOR $(95 \% \mathrm{Cl})$ & OR $(95 \% \mathrm{Cl})$ & AOR $(95 \% \mathrm{Cl})$ \\
\hline Age (continuous) & 1022 & $0.89(0.72-1.10)$ & $0.83(0.67-1.03)$ & $0.76(0.62-0.93)$ & $0.70(0.57-0.87)$ & $0.86(0.70-1.06)$ & $0.82(0.66-1.02)$ \\
\hline \multicolumn{8}{|l|}{ Sex } \\
\hline Boys & 481 & 1 & 1 & 1 & 1 & 1 & 1 \\
\hline Girls & 541 & $0.66(0.48-0.90)$ & $0.65(0.47-0.92)$ & $0.76(0.56-1.02)$ & $0.80(0.58-1.10)$ & $0.56(0.41-0.77)$ & $0.57(0.41-0.80)$ \\
\hline \multicolumn{8}{|l|}{ City of residence } \\
\hline Coimbra & 549 & 1 & 1 & 1 & 1 & 1 & 1 \\
\hline Lisbon & 352 & $1.74(1.24-2.43)$ & $1.75(1.23-2.50)$ & $1.49(1.08-2.06)$ & $1.55(1.11-2.18)$ & $2.07(1.48-2.90)$ & $2.16(1.52-3.09)$ \\
\hline Porto & 121 & $0.77(0.43-1.39)$ & $0.81(0.44-1.47)$ & $0.65(0.37-1.15)$ & $0.64(0.36-1.15)$ & $0.83(0.46-1.50)$ & $0.89(0.48-1.63)$ \\
\hline
\end{tabular}

Children BMI (IOTF cut-offs)

Normal
Overweight
Obesity

$776 \quad 1$

193

$1.19(0.79-1.77)$

53

$1.74(0.92-3.29)$

1

$0.94(0.61-1.45)$

1

$1.24(0.85-1.81)$

$1.32(0.69-2.54)$

\section{1}

$1.04(0.69-1.55)$

$0.87(0.43-1.77)$

\section{1}

$1.27(0.86-1.88)$

$1.09(0.54-2.23)$

1

$1.09(0.71-1.66)$ $0.80(0.36-1.75)$

Mother BMI (WHO cut-offs)

Normal

685

Obesity

246

91

1

Socioeconomic status

Low

High

250

376

$1.48(0.98-2.22)$

$1.22(0.76-1.94)$

$1.24(0.83-1.85)$

$396 \quad 1$

Sport Activity beyond school

Yes

739

283

No

$1.28(0.88-1.86)$

1

Self-Assessed Health

Excellent, Very good

Good, Bad, Very Bad 218

$804 \quad 1$

Mother DASS Depression score

$\begin{array}{cc}\text { T1 } & 514 \\ \text { T2 } & 201 \\ \text { T3 } & 307 \\ \text { Mother DASS Anxiety score } \\ \text { T1 } & 572 \\ \text { T2 } & 206 \\ \text { T3 } & 244\end{array}$

Mother DASS Stress score

$\begin{array}{ll}\text { T1 } & 415 \\ \text { T2 } & 311 \\ \text { T3 } & 296\end{array}$

$\begin{array}{lll}514 & 1 & 1 \\ 201 & 1.37(0.89-2.09) & 1.38(0.89-2.14) \\ 307 & 1.65(1.15-2.36) & 1.67(1.14-2.44)\end{array}$

DASS-C Depression, Anxiety and Stress scale - Children version, BMI Body Mass Index; IOTF International Obesity Taskforce, WHO World Health Organization: T1 T2, T3 - Tertiles 1, 2 and 3 of the DASS subscale score distribution 
mother-child depressive symptoms [51]. Other unmeasured factors may also play a role in the relation between motherchild mental health symptoms, namely poor parent-child attachment or inadequate supervision. These factors would be better explored using a longitudinal design [52].

\section{Strengths and limitations}

The appropriateness of assessing depression, anxiety and stress symptoms among children, specifically using the DASS-C, has been questioned, since these symptoms might be indistinguishable in children and adolescents [53]. In the scales' original psychometric assessment [33], only the stress subscale was pointed out as needing refinement. The Portuguese validation of the children version of the scale was conducted with a smaller $(n=361)$ sample of children slightly older compared to the children enrolled in our study (children aged 8 to 15 years old). In the validation study, the scale was individually filled, although administration occurred in collective and individual sessions [34], and lower correlations were also found for the stress subscale [34]. In this validation study, no differences in the results were found when the sample was stratified by age in younger (8-11 years) and older (12-15 years) children [34]. In the present study the factorial analysis of the DASS and the DASS-C suggests confirmation of the 3-factor solution for both versions (Supplementary material). Nevertheless, future studies should test the scale's reliability since few studies have documented this scale's performance in younger samples. Poor psychometric characteristics have also been found, particularly for the Anxiety and Stress subscales of the DASS-C [53]. For this reason, the results presented for these subscales should be interpreted with caution.

Assessing this type of variables in young children might be particularly difficult considering their literacy limitations, although possible with children aged 7 years and older and with a sixth-grade reading level or higher [54], which might have not been the case with the current sample. For this reason, the current results must also be interpreted with caution.

The current study did not explore the role of parental psychiatric condition as an associated factor with children's mental health symptoms, nor the history of any traumatic incident or other adverse childhood events, including child maltreatment [55]. Since the tertiles of the distribution of mothers' DASS scores were used as an exposure measure, and given the skewed distribution of such scores, these results show the probability of children declaring more symptomatology even for very low scores of mother's symptoms.

A major strength of the current study lies in children's self-reported outcome measure: children answered the DASS-C at school without parental influence. Previous research assessing mental health of primary school-aged children documents parental reports to specific questionnaires and not actual child answers, which are harder to obtain. In addition, parental reports of children mental health status may be biased, particularly, if mothers are experiencing depression [49].

Finally, due to the differences observed between included and excluded children (more girls, slightly older, more frequently from Coimbra and more frequently classified with high socioeconomic status were included), it is not possible to state that this group of children constitutes a representative sample of Portuguese schoolaged children and, for this reason, the results should not be generalized.

\section{Conclusion}

The potential negative developmental consequences associated with childhood depressive, anxiety and stress symptoms should be prevented. This study suggests that place of residence, individual characteristics and parental factors, in specific the depressive, anxiety and stress symptoms of the mother, are important modifiable factors that must be considered in the development of contextualized preventive efforts for childhood mental problems.

\section{Supplementary information}

Supplementary information accompanies this paper at https://doi.org/10. 1186/s12888-020-02498-z.

Additional file 1: Table S1. Principal component analysis: total variance explained for DASS and DASS-C. Table S2. Rotated Component Matrix (Varimax) for the DASS and DASS-C from Principal Component Analysis. Table S3. Cronbach alphas for DASS and DASS-C. Table S4. Characteristics of Included vs. Excluded children in analysis.

\section{Abbreviations}

AOR: Adjusted Odds Ratio; BMI: Body Mass Index; Cl: Confidence Interval; CNPD: Comissão Nacional de Proteção de Dados; DASS: Depression, Anxiety and Stress Scale; DASS-C: Depression, Anxiety and Stress Scale - Children; IOTF: International Obesity Task force; IQR: Interquartile Range; OR: Odds Ratio; Q1, Q2, Q3, Q4: Quartiles 1, 2, 3, 4; SES: Socioeconomic Status

\section{Acknowledgements}

We thank all the Directors of the "Agrupamentos Escolares" (schools) who accepted to participate in this project, the teachers directly involved and the parents of the children who authorized the questionnaires and anthropometric measures.

\section{Authors' contributions}

DC conceived the study aim, analyzed and interpreted the data and drafted the article. MC, CF, AG, AMMR, VRM, HN, and MRGS contributed to the study design and critically revised the article for important intellectual content. CP was responsible for the study design, acquisition of data and drafting the article. All authors approved the final manuscript as submitted and agree to be accountable for all aspects of the work.

\section{Funding}

This study was supported by a grant from Fundação para a Ciência e Tecnologia, Portugal, reference: PTDC/DTP-SAP/1520/2014. Projeto cofinanciado pelo COMPETE 2020, Portugal 2020 - Programa Operacional Competitividade e Internacionalização (POCI), União Europeia através do Fundo Europeu de Desenvolvimento Regional (FEDER) e Fundação para a Ciência e Tecnologia (FCT). The funders had no role in study design, data collection and analysis, decision to publish, or preparation of the manuscript. 


\section{Availability of data and materials}

The datasets used and/or analysed during the current study are available from the corresponding author on reasonable request.

\section{Ethics approval and consent to participate}

The study protocol was approved by Direção Geral do Ensino (Portuguese Ministry of Education) and Comissão Nacional de Proteção de Dados (CNPD), the Portuguese Data Protection Authority. All procedures were in accordance with the ethical standards of the Portuguese Data Protection Authority (Authorization number 745/2017) and with the 1964 Helsinki declaration and its later amendments or comparable ethical standards. Informed consent was obtained from children's parents.

\section{Consent for publication}

Not applicable.

\section{Competing interests}

The authors declare that they have no competing interests.

\section{Author details}

${ }^{1}$ Research Centre for Anthropology and Health, Department of Life Sciences, University of Coimbra, Calçada Martim de Freitas, Edifício São Bento, 3000-456 Coimbra, Portugal. ${ }^{2}$ CINEICC - Centre for Research in Neuropsychology and Cognitive and Behavioral Intervention, Faculty of Psychology and Educational Sciences, University of Coimbra, Coimbra, Portugal. ${ }^{3}$ Department of Animal Biology, Faculty of Sciences, University of Lisbon, Lisboa, Portugal. ${ }^{4}$ High School of Education, Polytechnic Institute of Viseu, Viseu, Portugal. ${ }^{5}$ Faculty of Human Kinetics, University of Lisbon, Lisboa, Portugal. ' Department of Geography and Tourism, University of Coimbra, Coimbra, Portugal. ${ }^{7}$ Faculty of Health Sciences, University Fernando Pessoa, Porto, Portugal.

\section{Received: 7 October 2019 Accepted: 14 February 2020}

Published online: 27 February 2020

\section{References}

1. Kieling C, Baker-Henningham H, Belfer M, Conti G, Ertem I, Omigbodun O, et al. Child and adolescent mental health worldwide: evidence for action. Lancet. 2011;378(9801):1515-25.

2. Polanczyk GV, Salum GA, Sugaya LS, Caye A, Rohde LA. Annual research review: a meta-analysis of the worldwide prevalence of mental disorders in children and adolescents. J Child Psychol Psychiatry. 2015;56(3):345-65.

3. Kovess-Masfety V, Van Engelen J, Stone L, Otten R, Carta MG, Biftoi A, et al. Unmet need for specialty mental health services among children across Europe. Psychiatr Serv. 2017;68(8):789-95.

4. Augusto GF. Mental health in Portugal in times of austerity. Lancet Psychiatry. 2014;1:109-10.

5. Kovess V, Carta MG, Pez O, Bitfoi A, Koç C, Goelitz D, et al. The school children mental health in Europe (SCMHE) project: design and first results. Clin Pract Epidemiol Ment Health. 2015;11(Suppl 1 M7):113-23.

6. Cartwright-Hatton S, McNicol K, Doubleday E. Anxiety in a neglected population: prevalence of anxiety disorders in pre-adolescent children. Clin Psychol Rev. 2006;26(7):817-33.

7. Martinsen KD, Neumer S-P, Holen S, Waaktaar T, Sund AM, Kendall PC. Selfreported quality of life and self-esteem in sad and anxious school children. BMC Psychology. 2016;4(1):45.

8. Fergusson DM, Woodward $\sqcup$. Mental health, educational, and social role outcomes of adolescents with depression. Arch Gen Psychiatry. 2002;59(3):225-31.

9. Kovacs M, Lopez-Duran N. Prodromal symptoms and atypical affectivity as predictors of major depression in juveniles: implications for prevention. J Child Psychol Psychiatry. 2010;51(4):472-96.

10. Kim-Cohen J, Caspi A, Moffitt TE, Harrington H, Milne BJ, Poulton R. Prior juvenile diagnoses in adults with mental disorder: developmental followback of a prospective-longitudinal cohort. Arch Gen Psychiatry. 2003;60(7): 709-17.

11. Bittner A, Egger HL, Erkanli A, Costelo EJ, Foley DL, Angold A. What do childhood anxiety disorders predict? J Child Psychol Psychiatry. 2007:48(12): 1174-83.

12. Birmaher B, Arbelaez C, Brent D. Course and outcome of child and adolescent major depressive disorder. Child Adolesc Psychiatr Clin N Am. 2002;11(3):619-37.
13. Paananen R, Ristikari T, Merikukka M, Gissler M. Social determinants of mental health: a Finnish nationwide follow-up study on mental disorders. J Epidemiol Community Health. 2013;67(12):1025-31.

14. Reiss F. Socioeconomic inequalities and mental health problems in children and adolescents: a systematic review. Soc Sci Med. 2013;90:24-31.

15. Stenmark H, Bergström E, Hägglöf B, Öhman A, Petersen S. Mental problems and their socio-demographic determinants in young schoolchildren in Sweden, a country with high gender and income equality. Scand J Public Health. 2016;44(1):18-26.

16. Melchior M, Chastang J-F, Walburg V, Arseneault L, Galéra C, Fombonne E. Family income and youths' symptoms of depression and anxiety: a longitudinal study of the French GAZEL youth cohort. Depress Anxiety. 2010;27(12):1095-103.

17. Apter G, Bobin A, Genet M-C, Gratier M, Devouche E. Update on mental health of infants and children of parents affected with mental health issues. Curr Psychiatry Rep. 2017;19(10):72.

18. Barker ED, Copeland W, Maughan B, Jaffee SR, Uher R. Relative impact of maternal depression and associated risk factors on offspring psychopathology. Br J Psychiatry. 2012;200(2):124-9.

19. Waugh CE, Muhtadie L, Thompson RJ, Joormann J, Gotlib $H$. Affective and physiological responses to stress in girls at elevated risk for depression. Dev Psychopathol. 2012;24(2):661-75.

20. Sutaria S, Devakumar D, Yasuda SS, Das S, Saxena S. Is obesity associated with depression in children? Systematic review and meta-analysis. Arch Dis Child. 2018:104(1):64-74.

21. van Geel M, Vedder P, Tanilon J. Are overweight and obese youths more often bullied by their peers? A meta-analysis on the relation between weight status and bullying. Int J Obes (Lond). 2014:38(10):1263-7.

22. Ahmadpanah M, Nazaribadie M, Mohammadi MR, Hooshyari Z, Alavi SS, Ghaleiha A, et al. The prevalence of psychiatric disorders in children and adolescents in Hamadan Province, west of Iran. J Res Health Sci. 2018;18(4):e00432.

23. Magiati I, Ponniah K, Ooi YP, Chan YH, Fung D, Woo B. Self-reported depression and anxiety symptoms in school-aged Singaporean children. Asia Pac Psychiatry. 2015;7(1):91-104.

24. Schuch FB, Vancampfort D, Firth J, Rosenbaum S, Ward PB, Silva ES, et al. Physical activity and incident depression: a meta-analysis of prospective cohort studies. Am J Psychiatry. 2018;175(7):631-48.

25. Opdal IM, Morseth B, Handegård BH, Lillevoll K, Ask H, Nielsen CS, et al. Change in physical activity is not associated with change in mental distress among adolescents: the Tromsø study: fit futures. BMC Public Health. 2019; 19(1):916.

26. Belfer ML. Child and adolescent mental disorders: the magnitude of the problem across the globe. J Child Psychol Psychiatry. 2008;49(3):226-36.

27. Padez C, Fernandes T, Mourão I, Moreira P, Rosado V. Prevalence of overweight and obesity in 7-9-year-old Portuguese children: trends in body mass index from 1970-2002. Am J Hum Biol. 2004;16(6):670-8.

28. Machado-Rodrigues AM, Fernandes R, Gama A, Mourão I, Nogueira H, Rosado-Marques $V$, et al. The association of irregular sleep habits with the risk of being overweight/obese in a sample of Portuguese children aged 69 years. Am J Hum Biol. 2018;30(4):e23126.

29. Pais-Ribeiro JL, Honrado A, Leal I. Contribuição Para o Estudo da Adaptação Portuguesa das Escalas de ansiedade, Depressão e stress (EADS) de 21 Itens de Lovibond e Lovibond. Psicologia, Saúde \& Doenças. 2004;5:229-39.

30. Lovibond $\mathrm{P}$, Lovibond $\mathrm{S}$. The structure of negative emotional states: comparison of the depression anxiety stress scales (DASS) with the Beck depression and anxiety inventories. Behav Res Ther. 1995;33(3):335-43.

31. Cole TJ, Bellizzi MC, Flegal KM, Dietz WH. Establishing a standard definition for child overweight and obesity worldwide: international survey. Br Med J. 2000;320(7244):1240-3.

32. Ravens-Sieberer U, Auquier P, Erhart M, Gosch A, Rajmil L, Bruil J, et al. The KIDSCREEN-27 quality of life measure for children and adolescents: psychometric results from a cross-cultural survey in 13 European countries. Qual Life Res. 2007;16(8):1347-56.

33. Szabó M, Lovibond PF. Anxiety, depression and tension/stress in children. J Psychopathol Behav Assess. 2006;28(3):192-202.

34. Leal IP, Antunes R, Passos T, Pais-Ribeiro JL, Maroco J. Estudo da Escala de Depressão, ansiedade e Stresse Para Crianças (EADS-C). Psicologia, Saúde \& Doenças. 2009;10:277-84.

35. Chaplin TM, Aldao A. Gender differences in emotion expression in children: a meta-analytic review. Psychol Bull. 2013;139(4):735-65. 
36. Silver E, Mulvey EP, Swanson JW. Neighborhood structural characteristics and mental disorder: Faris and Dunham revisited. Soc Sci Med. 2002;55(8):1457-70.

37. Wang Y, Min J, Khuri J, Li M. A systematic examination of the association between parental and child obesity across countries. Adv Nutr. 2017:8(3):436-48.

38. Voerman E, Santos S, Patro Golab B, Amiano P, Ballester F, Barros H, et al. Maternal body mass index, gestational weight gain, and the risk of overweight and obesity across childhood: an individual participant data meta-analysis. PLoS Med. 2019;16(2):e1002744.

39. Güngör NK. Overweight and obesity in children and adolescents. J Clin Res Pediatr Endocrinol. 2014;6(3):129-43.

40. Poulton R, Caspi A, Milne BJ, Thomson WM, Taylor A, Sears MR, et al, Association between children's experience of socioeconomic disadvantage and adult health: a life-course study. Lancet. 2002;360(9346):1640-5.

41. White RL, Babic MJ, Parker PD, Lubans DR, Astell-Burt T, Lonsdale C. Domain-specific physical activity and mental health: a meta-analysis. Am J Prev Med. 2017;52(5):653-66.

42. Lubans D, Richards J, Hillman C, Faulkner G, Beauchamp M, Nilsson M, et al. Physical activity for cognitive and mental health in youth: a systematic review of mechanisms. Pediatrics. 2016;138(3):e20161642.

43. Tsai $\mathrm{CL}$, Wang $\mathrm{CH}$, Pan $\mathrm{CY}$, Chen FC, Huang TH, Chou FY. Executive function and endocrinological responses to acute resistance exercise. Front Behav Neurosci. 2014:8:262.

44. Lane AM, Lovejoy DJ. The effects of exercise on mood changes: the moderating effect of depressed mood. J Sports Med Phys Fitness. 2001; 41(4):539-45.

45. Chaput J-P, Barnes JD, Tremblay MS, Fogelholm M, Hu G, Lambert EV, et al. Inequality in physical activity, sedentary behaviour, sleep duration and risk of obesity in children: a 12-country study. Obes Sci Pract. 2018;4(3):229-37.

46. Wang Y, Dix T. Mothers' depressive symptoms and children's externalizing behavior: Children's negative emotionality in the development of hostile attributions. J Fam Psychol. 2017;31(2):214-23.

47. Downey G, Coyne JC. Children of depressed parents: an integrative review. Psychol Bull. 1990;108(1):50-76.

48. Webster KD, de Bruyn MM, Zalwango SK, Sikorskii A, Barkin JL, FamiliarLopez I, et al. Caregiver socioemotional health as a determinant of child well-being in school-aged and adolescent Ugandan children with and without perinatal HIV exposure. Trop Med Int Health. 2019;24(5):608-19.

49. Ordway MR. Depressed mothers as informants on child behavior: methodological issues. Res Nurs Health. 2011;34(6):520-32.

50. Goodman SH, Gotlib IH. Risk for psychopathology in the children of depressed mothers: a developmental model for understanding mechanisms of transmission. Psychol Rev. 1999;106(3):458-90.

51. Dix T, Yan N. Mothers' depressive symptoms and infant negative emotionality in the prediction of child adjustment at age 3: testing the maternal reactivity and child vulnerability hypotheses. Dev Psychopathol. 2014;26(01):111-24.

52. Hyland P, Shevlin M, Elklit A, Christoffersen M, Murphy J. Social, familial and psychological risk factors for mood and anxiety disorders in childhood and early adulthood: a birth cohort study using the Danish registry system. Soc Psychiatry Psychiatr Epidemiol. 2016;51(3):331-8.

53. Patrick J, Dyck M, Bramston P. Depression anxiety stress scale: is it valid for children and adolescents? J Clin Psychol. 2010;66(9):996-1007.

54. Sharp L, Lipsky M. Screening for depression across the lifespan: a review of measures for use in primary care settings. Am Fam Physician. 2002;66(6):1001-8.

55. Felitti VJ, Anda RF, Nordenberg D, Williamson DF, Spitz AM, Edwards, et al. Relationship of childhood abuse and household dysfunction to many of the leading causes of death in adults. The adverse childhood experiences (ACE) study. Am J Prev Med. 1998;14(4):245-58.

\section{Publisher's Note}

Springer Nature remains neutral with regard to jurisdictional claims in published maps and institutional affiliations.

Ready to submit your research? Choose BMC and benefit from:

- fast, convenient online submission

- thorough peer review by experienced researchers in your field

- rapid publication on acceptance

- support for research data, including large and complex data types

- gold Open Access which fosters wider collaboration and increased citations

- maximum visibility for your research: over $100 \mathrm{M}$ website views per year

At BMC, research is always in progress.

Learn more biomedcentral.com/submissions 